\title{
Wheat Genetic Resources in Nepal
}

\author{
Bal K. Joshi*, Ashok Mudwari and Madan R. Bhatta \\ NARC, PO Box 1135 Kathmandu, Nepal
}

\begin{abstract}
Genetic diversity must be maintained and utilized for sustainable agriculture development. The amount of genetic diversity in the country depends on the number and diversity of the original ancestors involved in the creation of a germplasm pool, wild relatives and existing landraces. The objective of this research was to study the diversity of wheat gene pool present in the Nepalese bread wheat cultivars and landraces that could help for developing conservation and utilization strategy effectively. We examined the pedigrees of 35 Nepalese wheat cultivars and surveyed the literature for distribution of landraces and wild relatives of wheat. Cultivated landraces of spring and winter type, wild landraces and diploid species of wheat are found in Nepal. There are 35 improved wheat cultivars, 540 landraces and 10 wild relatives of wheat. Crosses between winter and spring wheat gene pools are far more common and offer a new source of diversity. Mexico, India and Nepal are the origin countries for 35 cultivars. In Nepal four cultivars were bred and developed using foreign landraces and maximum number of cultivars was developed in Mexico. Lerma 52, first improved cereal variety to be released in the history of cereal breeding in Nepal was released in 1960. A total of 89 ancestors originated in 22 different countries were used to develop these cultivars. Highest number of ancestors was from India. Ancestors of both aestivum and durum species having winter, spring and intermediate growth habit indicated the collection of wide gene pool. Most of the ancestors were aestivum $(76.40 \%)$ and spring growth habit $(57.31 \%)$. Modern varieties are replacing the landraces and improved old varieties resulted in the genetic erosion. Therefore, in situ, on farm and ex situ conservations are necessary for maintaining these genetic variations. Unutilization of local landraces in breeding program may be the major factor that causes to accelerate the genetic erosion. Gene pool from these landraces along with international gene pool could make towards success in developing high yielding cultivars with wide adaptability. In this study, cultivars and landraces surveyed represent a wide range of variation for different areas of origin and adaptation.
\end{abstract}

Key words: Ancestor, landrace, origin, wheat gene pool

\section{INTRODUCTION}

Wheat has been growing since time immemorial particularly in Far and Mid Western hills of Nepal. Mudwari (1999) reported many landraces and 10 wild relatives of wheat in Nepal. It is the third most important crop after rice and maize in Nepal. During mid 1960s the yield potential of dwarf high yielding varieties initiated a scope for raising wheat production in the country. Several exotic varieties were obtained through CIMMYT and USAID (NARC 1997). National Wheat Development Programme was established in 1972 to organize the research and development works on wheat as a commodity crop. Since then, there have been great achievement brought out by the consolidated efforts of wheat researchers, extension workers and farmers. So far there are 35 improved wheat cultivars and $90 \%$ of the wheat area is covered by modern wheat cultivars in Nepal (Bhatta et al 2000). Currently wheat is mainly used for bread and biscuits and is becoming more important in Nepalese economy.

Genetic diversity is necessary to derive different transgenic segregants suitable for different agroecology to meet the needs of farmers. Both the potential for long term genetic gain and the reduction of genetic vulnerability may depend on the genetic diversity present in the genetic base. The amount of 
genetic diversity presents depends on the number and diversity of the original ancestors involved in the creation of a germplasm pool and existing landraces. The level of genetic variation present in gene pools of most important crops has been analyzed by studying the pedigree relationship between cultivars. Kinship coefficients estimation of cultivars of oat (Souza and Sorrells 1989), soybean (Cox et al 1985a), winter wheat (Cox et al 1985b), rice (Dilday 1990) and barley (Martin et al 1991) has shown that a restricted number of ancestral genotypes account for a large proportion of the variation present in released cultivars. However Nepalese wheat cultivars possess great diversity because of using many ancestral genotypes to develop them (Joshi et al 2004). Richness on wheat taking into account the ancestors of cultivars and landraces should be assessed for effective conservation and utilization of wheat gene pools. Therefore we have focused here on landraces and its distribution in Nepal and countries from where genes were introduced through improved lines in Nepal.

\section{METHODOLOGY}

Literature related to wheat exploration, improved cultivars and Nepalese landraces were reviewed. Two sites, National Wheat Research Program (NWRP), Bhairawa and Plant Genetic Resources (PGR) Unit, Khumaltar were visited. PGR Unit has collected many wheat landraces from different parts of Nepal. Based on collection data landraces distribution were indicated in Nepal map. Frequency of wheat accessions collected from different districts and conserved in PGR Unit were computed. We examined the pedigrees of all 35 cultivars. Altogether 35 cultivars had been released in Nepal from 1960 to 2001. Most of the cultivars were introduced either from CIMMYT, Mexico or India. The pedigrees of all bread wheat cultivars were traced back to their ancestors that had no known relationship each other. The source of pedigrees and release dates for cultivars were Jain (1994), NARC (1997), Bland (2001), Skovmand et al (1997) and Skovmand et al (2000). Countries from where the genes introduced through the improved lines in Nepal were located in world map.

\section{RESULTS AND DISCUSSION}

The land in Nepal has the largest variations in altitude in the world. It lies between $26^{\circ} 22^{\prime} \mathrm{N}$ to $30^{\circ} 27^{\prime} \mathrm{N}$ and $80^{\circ} 4^{\prime} \mathrm{E}$ to $88^{\circ} 12^{\prime} \mathrm{E}$. Elevation ranges from 60 to $8848 \mathrm{~m}$. Three types of land Bari (upland), Khet (lowland) and flat and fertile with good soil depths produce wheat indicating the diverse wheat genotypes adapted to different production environments. Wheat is grown as winter crop sown in October or November and harvested in March or April. It is also grown as a summer crop sown in April or May coming to fruition in September or October. Due to the varied agro-ecological diversity of the country, it is possible to plant same cultivar in both winter and summer season. Nepal is not original home for wheat but under the CGIAR system Nepal received a lot of wheat genotypes. In 1965, the Department of Agriculture launched a Grow more wheat campaign with the introduction of Mexican semi dwarf wheat resulted in a rapid expansion in wheat area and production.

Nepal is politically divided into five regions (Eastern, Central, Western, Mid Western and Far Western). Only two exploration missions have targeted wheat species in Western Nepal. Eighteen exploration programs were carried out in different parts of Nepal to collect different crops species. There are 63 landraces, which are differed by name and conserved in ABD (Table 1). These are collected from different altitude ranging from 720 to $3353 \mathrm{~m}$. Farmer unit of description (FUD) is generally related to their phenotype and use value. FUD also indicates the level of diversity present in wheat gene pool. There may be many landraces that are genetically same but differed only by name or vise versa. These need to verify. ABD has 390 accessions of wheat landraces collected from all over the Nepal. These are conserved ex situ at medium term storage facility in Khumaltar, Kathmandu. Due to agro ecological differences Nepalese genotypes may contain unique genes. Dutch scientists who collected in around 1981, wheat in remote part of the Himalayan in Nepal has found material that is new and considerably 
different from germplasm already in gene bank anywhere in the world (Hawkes 1981). These genes if conserved properly could be enough to fulfill wheat diversity demand for developing wheat cultivars for next century.

Table 1. Different Nepalese wheat landraces and their cultivating area

\begin{tabular}{|c|c|c|c|c|}
\hline SN & Landrace & District & Location & Altitude, $\mathrm{m}$ \\
\hline 1 & Badi gahun & Bajura & Gadukhati-9 & 1768 \\
\hline 2 & Bangali gahun & Kalikot & Jubitha-7, Jubitha & 1792 \\
\hline 3 & Bartole gahun & Baitadi & Patan & 1372 \\
\hline 4 & Bhabri gahun & Mugu & Srinagar-5, Chaina & 1960 \\
\hline 5 & Bhagere gahun & Baglung & Bhimpokhara & 1565 \\
\hline 6 & Bhartole gahun & Baitadi & Gokuleswor-1, Kalchunde & 720 \\
\hline 7 & Bhote gahun & Solukhumbu & Salleri & 2408 \\
\hline 8 & Bhugari gahu & Bajura & Atichaul-1 & 1981 \\
\hline 9 & Bikase gahun & Darchula & Gokuleswor-3, Gokuleswor & 750 \\
\hline 10 & Bikasi seto gahun & Sallyan & Dandagaon & 1200 \\
\hline 11 & Bugoti & Bajura & Dogdi-6 & 1829 \\
\hline 12 & Bungoli & Bajhang & Kalukheti-8 & 1737 \\
\hline 13 & Chamdi gahun & Bajura & Gadukhati-9 & 1768 \\
\hline 14 & Dabde gahun & Jumla & Patrasi-7, Shelagarhigaon & 2713 \\
\hline 15 & Dabdi gahun & Dandeldhura & Joishina & 1585 \\
\hline 16 & Dabdikhane gahun & Dandeldhura & Matar gaon & \\
\hline 17 & Dalkhane gahun & Kalikot & Mahadev-7, Sarkivada & 1980 \\
\hline 18 & Dapche gahun & Dandeldhura & Bhel & 1585 \\
\hline 19 & Daudi & Baitadi & Patan & 1372 \\
\hline 20 & Daudi gahun & Baitadi & Shidheswor-8, Amarkholi & 2070 \\
\hline 21 & Dhaule gahun & Baitadi & Gokuleswor-1, Kalchunde & 720 \\
\hline 22 & Dho & Mustang & Kagbeni & 2697 \\
\hline 23 & Dho/gahun & Mustang & Jharkot & 3353 \\
\hline 24 & Dhu & Mustang & Khinga & 3216 \\
\hline 25 & Dolkhe gahun & Khotang & Khalde & 1402 \\
\hline 26 & Dudhe murilo & Rukum & Vulma & 823 \\
\hline 27 & Gahun & Baglung & Dobira & 1010 \\
\hline 28 & Gaile gahun & Bajura & Gadukhati-9 & 1768 \\
\hline 29 & Geru gahun & Mugu & Pina-5, Balagaon & 2035 \\
\hline 30 & Gharelu gahun & Dandeldhura & Manara & 1158 \\
\hline 31 & Hansa gahun & Myagdi & Benibagar & 792 \\
\hline 32 & Jhirke & Bajhang & Majhigaon-8 & 1585 \\
\hline 33 & Jhuse gahun & Sallyan & Dandagaon & 1100 \\
\hline 34 & Jhuse rato gahun & Kalikot & Mahadev-7, Sarkivada & 1980 \\
\hline 35 & Keuma gahun & Solukhumbu & Chhulembu & 2195 \\
\hline 36 & Lal gahun & Kanchanpur & Mahendra nagar-19 & \\
\hline 37 & Lalitpur local & & & \\
\hline 38 & Lera rato gahun & Sallyan & Dandagaon & 1200 \\
\hline 39 & Lere seto gahun & Sallyan & Tharmare & 1160 \\
\hline 40 & Thaniya gahun & Jumla & Birat-9, Ludku & 2390 \\
\hline
\end{tabular}




\begin{tabular}{llllr}
\hline SN & Landrace & District & Location & Altitude, m \\
\hline 41 & Lunthung gahun & Taplejung & Lelep-5, Lunthum & 1800 \\
42 & Mude gahun & Dandeldhura & Bhandare & 1530 \\
43 & Mudlo gahun & Baglung & Gitapatha & 1094 \\
44 & Mudule gahun & Arghakhanchi & Dhikura-2, Gairakot & 1200 \\
45 & Mudulo gahun & Myagdi & Dhode & 747 \\
46 & Murala gahun & Rolpa & Khumil & 1500 \\
47 & Murulo rato gahun & Jajarkot & Gagane khola & 2775 \\
48 & Nano gahun & Dandeldhura & Ghatal & 1768 \\
49 & Paude gahun & Baitadi & Vasling-3, Gwane & 2040 \\
50 & Pawai & Bajura & Kolti & 2000 \\
& & & & \\
51 & Peta gahun & Solukhumbu & Chhulembu & 2195 \\
52 & Rani gahu & Bajhang & Majhigaon-8 & 1585 \\
53 & Rato gahun & Jajarkot & Risang & 2850 \\
54 & Ratonal & Baitadi & Patan & 1372 \\
55 & Ratonale & Baitadi & Patan-1 & 1372 \\
56 & Rupali gahun & Dandeldhura & Amargadhi-2, Dotighatal & 1750 \\
57 & Sano gahun & & Suda & \\
58 & Sate gahun & Taplejung & Nankholyang-5, Myakha & 900 \\
59 & Seto gahun & Rukum & Khara & \\
60 & Talak gahu & & Mahendra nagar & 1768 \\
61 & Tari gaire & Bajura & Gadukhati-9 & 1768 \\
62 & Those gahun & Bajura & Gadukhati-9 & 2750 \\
63 & Thulo ghumche & Jajarkot & Danda gaon & \\
\hline
\end{tabular}

Source: Gupta et al 2000.

Nepal has many locally adapted wheat but any one has not been used or improved for developing Nepalese wheat cultivars. Such trends lead to genetic erosion. NWRP has maintained 150 landraces and ABD has 390 accessions of wheat. There may be duplicate accessions in these two places. Removing duplicate accessions and adding new one should be made continuously. Characterization, evaluation and utilization of these landraces in breeding program by NWRP and ABD could certainly enhance the wheat genes pool conservation. Landraces are mostly found in Western regions and only few locations of Central and Eastern regions (Figure 1) in Nepal. It is still unanswered why these landraces get popularized in remote areas e.g. Western Nepal. There are many important landraces in Western region. Dabad Khani is most popular local wheat suited to maize based cropping pattern in Western region. This distribution pattern could help to locate diverse wheat areas and to implement in situ, on farm conservation and exploration program. This pattern suggests that there are diverse wheat landraces adapted to different environments.

ABD has representative wheat landraces from 29 out of total 75 districts of Nepal (Table 2). Highest number of wheat accessions was collected from Dandeldhura (3.59\% of total accessions) followed by Baitadi (3.33\%), Bajura and Baglung. Dandeldhura, Baitadi, Bajura, Baglung and Achham may be the focal area in term of wheat landraces diversity. Due to the expansion of modern varieties these landraces are under threats of extinction. Extensive survey and duplication study of accessions could help to control genetic erosions and conservation cost. Many of these landraces are still grown by farmers and are the mixtures of white and brown coloured spike, amber and red coloured kernels, awned and awnless characters. These landraces have a wide range of natural adaptation to withstand varied abiotic and biotic conditions. In addition, these landraces have high tillering ability, withstand severe drought stress, have high protein content and longer seed dormancy. These are pure spring bread wheat types. 
Some landraces with winter growth type have been reported in the northern high mountain area bordering Tibet, China. In addition to these, some diploid species have also been reported in the northern high mountains. Since these landraces are adapted to a small confined area, and wheat was considered a minor cereal until the middle of $20^{\text {th }}$ century, there was no attempt to improve these landraces or the production practices. These landraces are generally grown under marginal lands under rainfed and low fertility conditions.

Table 2. Frequency and total accessions of wheat landraces collected from different districts of Nepal and conserved in Agriculture Botany Division Khumaltar

\begin{tabular}{llrrrlrr}
\hline SN & District & Accession, $\mathrm{n}$ & Frequency, $\%$ & SN & District & Accession, $\mathrm{n}$ & Frequency, $\%$ \\
\hline 1 & Achham & 11 & 2.82 & 17 & Khotang & 3 & 0.77 \\
2 & Arghakhanchi & 1 & 0.26 & 18 & Manang & 5 & 1.28 \\
3 & Baglung & 12 & 3.08 & 19 & Mugu & 5 & 1.28 \\
4 & Baitadi & 13 & 3.33 & 20 & Mustang & 9 & 2.31 \\
5 & Bajhang & 6 & 1.54 & 21 & Myagdi & 6 & 1.54 \\
6 & Bajura & 12 & 3.08 & 22 & Nawalparasi & 1 & 0.26 \\
7 & Dandeldhura & 14 & 3.59 & 23 & Panchthar & 1 & 0.26 \\
8 & Darchula & 4 & 1.03 & 24 & Pyuthan & 2 & 0.51 \\
9 & Doti & 6 & 1.54 & 25 & Rolpa & 2 & 0.51 \\
10 & Gorkha & 1 & 0.26 & 26 & Rukum & 6 & 1.54 \\
& & & & & & & \\
11 & Gulmi & 2 & 0.51 & 27 & Sallyan & 5 & 1.28 \\
12 & Jajarkot & 5 & 1.28 & 28 & Solukhumbu & 7 & 1.79 \\
13 & Jumla & 6 & 1.54 & 29 & Taplejung & 2 & 0.51 \\
14 & Kalikot & 5 & 1.28 & 30 & Unknown & 236 & 60.51 \\
15 & Kanchanpur & 1 & 0.26 & & & & 390 \\
16 & Kaski & 1 & 0.26 & & Total & \\
\hline
\end{tabular}

Thirty-five improved bread wheat varieties suitable to hills, plains and Western regions of Nepal are real efforts of researchers to be released during the period from 1960 to 2001 . More numbers of crosses involving many parental lines in cultivars like Annapurna 2, Annapurna 4, Bhrikuti, LR64, RR21, NP884 and NP809 indicate the effort of scientists to collect value genes in single genotype. Four cultivars were released in 1997, which is the year of releasing highest number of cultivars. These cultivars were Achyut, Kanti, Pasang Lhamu and Rohini. Lerma 52, first improved cereal variety to be released in the history of cereal breeding in Nepal (Bland 2001) was released in 1960.

A total of 89 ancestors originated in 22 different countries were used to develop 35 cultivars (Table 4). Mexico, India and Nepal are the origin countries for 35 cultivars. In Nepal four cultivars had been originated and maximum number of cultivars was originated in Mexico. Ancestors of both aestivum and durum species having winter, spring and intermediate growth habit indicated the collection of wide gene pool. 
Table 3. Improved bread wheat varieties released from 1960 to 2001 in Nepal

\begin{tabular}{|c|c|c|c|c|c|c|}
\hline $\mathrm{SN}$ & Variety & Abbr $\dagger$ & Pedigree & Origin & $\begin{array}{l}\text { Year } \\
\text { released }\end{array}$ & $\begin{array}{c}\text { Area of } \\
\text { adaptation }\end{array}$ \\
\hline 1. & Achyut & $\mathrm{ACH}$ & CPAN168/HD2204 & India & 1997 & Plains \\
\hline 2. & Annapurna1 & ANNA1 & KVZ/BUHO//KAL/BB & Mexico & 1988 & Hills \\
\hline 3. & Annapurna2 & ANNA2 & NAPO/TOB//8156/3/KAL/BB & India & 1988 & Hills \\
\hline 4. & Annapurna3 & ANNA3 & KVZ/BUHO//KAL/BB & Mexico & 1991 & Hills \\
\hline 5. & Annapurna4 & ANNA4 & $\begin{array}{l}\text { KVZ/3/CC/INIA//CNO/ELGAU/4/S } \\
\text { N64 }\end{array}$ & Mexico & 1994 & Hills \\
\hline 6. & Bhrikuti & $\mathrm{BK}$ & CMT/COC75/3/PLO//FURY/ANA & Mexico & 1994 & Plains \\
\hline 7. & $\begin{array}{l}\text { Bhairahawa } \\
\text { Line1022 }\end{array}$ & BL1022 & PVN/ALD & Nepal & 1991 & Western Terai \\
\hline 8. & $\begin{array}{l}\text { Bhairahawa } \\
\text { Line1135 }\end{array}$ & BL1135 & QTZ/TAN & Nepal & 1994 & Plains \\
\hline 9. & $\begin{array}{l}\text { Bhairahawa } \\
\text { Line1473 }\end{array}$ & BL1473 & NL297/NL352 & Nepal & 1999 & Plain \& Hills \\
\hline 10. & $\begin{array}{l}\text { Hybrid } \\
\text { Delhi1982 }\end{array}$ & HD1982 & E5557/HD845 & India & 1975 & Western Plains \\
\hline 11. & Kalyansona & KAL & PJ/GB55 & Mexico & 1968 & Plains \\
\hline 12. & Kanti & KANTI & LIRA/FFN//VEE & Mexico & 1997 & Hills \\
\hline 13. & Kenya291 & K291 & $\mathrm{NA} \ddagger$ & Kenya & 1962 & Hills \\
\hline 14. & Lerma52 & L52 & MTA/K324 & Mexico & 1960 & Hills \\
\hline 15. & Lerma Rojo64 & LR64 & Y50/N10B//L52/3/2*LR & Mexico & 1967 & Hills \\
\hline 16. & Lumbini & LUM & $\mathrm{E} 4871 / \mathrm{PJ}$ & India & 1981 & Plains \\
\hline 17. & Nepal Line251 & NL251 & WH147/HD2160//2*WH147 & India & 1988 & Plains \\
\hline 18. & Nepal Line297 & NL297 & HD2137/HD2186//HD2160 & India & 1985 & Plains \\
\hline 19. & Nepal Line30 & NL30 & HD832/BB & India & 1975 & Western Plains \\
\hline 20. & New Pusa799 & NP799 & NP 792 & India & 1962 & Hills \\
\hline 21. & New Pusa809 & NP809 & DO/C518//SPP/NP114/3/WIS245 & India & 1962 & Hills \\
\hline 22. & New Pusa835 & NP835 & NP760/RN & India & 1962 & Plains \\
\hline 23. & New Pusa852 & NP852 & $\mathrm{KF} / 2 * \mathrm{NP} 761$ & India & 1962 & Plains \\
\hline 24. & New Pusa884 & NP884 & $\begin{array}{l}\text { KC6042/GUL//PLT/3/K58/N/4/NP7 } \\
55\end{array}$ & India & $\mathrm{NA} \dagger$ & Plains \\
\hline 25. & Pasang Lhamu & PAL & PGO/SERI & Mexico & 1997 & Hills \\
\hline 26. & Pitic62 & PI & YT54/N10B 26.1C & Mexico & 1967 & Hills \\
\hline 27. & Rohini & $\mathrm{ROH}$ & PRL/TONI//CHIL & Nepal & 1997 & Plains \\
\hline 28. & Rust Resistant 21 & RR21 & $\begin{array}{l}\text { II53.388/AN/3/YT54/N10B/3/LR/4/ } \\
\text { B4946.A.4.18.2.IY/Y53//3*Y50 }\end{array}$ & Mexico & 1971 & Hills \& Plains \\
\hline 29. & S331 & S331 & LR64/HUAR & Mexico & 1971 & Hills \& Plains \\
\hline 30. & Siddhartha & SID & HD2092/HD1962//E4870/3/K65 & India & 1983 & Plains \\
\hline 31. & Sonora64 & SN64 & YT54/N10B//2*Y54 & Mexico & 1967 & Hills \\
\hline 32. & Triveni & TRI & HD1963/HD1931 & India & 1982 & Plains \\
\hline 33. & Uttar Pradesh262 & UP262 & S 308/BAJIO 66 & India & 1978 & Plains \\
\hline 34. & Vaskar & VKR & TZPP/PL//7C & Mexico & 1983 & $\begin{array}{l}\text { Mid-western } \\
\text { Plains }\end{array}$ \\
\hline 35. & Vinayak & VIN & LC55 & India & 1983 & Plains \\
\hline
\end{tabular}

$\dagger$ Abbr, Abbreviation. $\$$ NA, Not available.

Source: NARC 1997.

Wheat genes from all regions of the world were introduced in Nepal through improved cultivars (Figure 2). Maximum ancestors were from India followed by USA and Kenya. Thanks to CGIAR, India, USA, Kenya, Australia and Argentina. Involvement of ancestors from 22 countries indicates the introduction of genes adapted to different geographic locations. Even though contributions of Nepalese wheat landraces in the world is not known, world contribution is recognized in wheat development in Nepal. A single landrace of each of eight countries have been used in developing wheat cultivars probably because of having value genes with them. It can be concluded that breeders can develop best varieties by reshuffling the genes from these wide collections. 
Table 4. Ancestors of 35 Nepalese wheat cultivars and their origin

\begin{tabular}{|c|c|c|c|c|c|c|}
\hline \multirow[t]{2}{*}{ SN } & \multirow[t]{2}{*}{ Name } & \multirow[t]{2}{*}{$\mathrm{Abb} \dagger$} & \multicolumn{2}{|l|}{ Origin } & \multirow[t]{2}{*}{ Growth habit } & \multirow[t]{2}{*}{ Species } \\
\hline & & & Name & Abb & & \\
\hline 1. & 21931 & 21931 & ISREAL & ISL & $?$ & $\overline{\text { AESTIVUM }}$ \\
\hline 2. & 36896 & 36896 & ARGENTINA & ARG & $?$ & AESTIVUM \\
\hline 3. & $8 \mathrm{~A}$ & $8 \mathrm{~A}$ & INDIA & IND & $?$ & AESTIVUM \\
\hline 4. & $8 \mathrm{~B}$ & $8 \mathrm{~B}$ & INDIA & IND & ? & $?$ \\
\hline 5. & $9 \mathrm{D}$ & $9 \mathrm{D}$ & INDIA & IND & $?$ & AESTIVUM \\
\hline 6. & AKAGOMUGHI & AGM & JAPAN & JPN & WINTER & AESTIVUM \\
\hline 7. & ALFREDO CHAVES 6.21 & $\mathrm{AC}$ & BRAZIL & BRA & SPRING & AESTIVUM \\
\hline 8. & B4946.A.4.18.2.IY & B4946 & $?$ & $?$ & $?$ & $?$ \\
\hline 9. & BONZA & BZA & COLOMBIA & COL & SPRING & AESTIVUM \\
\hline 10. & BREVOR & BVR & USA & USA & WINTER & AESTIVUM \\
\hline 11. & BUNGE NO 2 & $\mathrm{BN} 2$ & $?$ & ? & $?$ & ? \\
\hline 12. & BUTTON & BUTTON & $?$ & $?$ & $?$ & AESTIVUM \\
\hline 13. & $\mathrm{C} 13$ & $\mathrm{C} 13$ & INDIA & IND & SPRING & AESTIVUM \\
\hline 14. & C209 & C209 & INDIA & IND & SPRING & AESTIVUM \\
\hline 15. & CARIANCA422 & CAR422 & CHILE & CHL & WINTER & AESTIVUM \\
\hline 16. & CENTENARIO & CTR & BRAZIL & BRA & SPRING & AESTIVUM \\
\hline 17. & CHRIS & CHR & USA & USA & SPRING & AESTIVUM \\
\hline 18. & CLEMENT & CMT & NETHERLANDS & NLD & WINTER & AESTIVUM \\
\hline 19. & CPAN1687 & CPAN1687 & INDIA & IND & SPRING & AESTIVUM \\
\hline 20. & DAVIS6301 & D6301 & USA & USA & $?$ & AESTIVUM \\
\hline 21. & DEMOCRATE & DO & USA & USA & ? & AESTIVUM \\
\hline 22. & EL GAUCHO & ELGAU & ARGENTINA & ARG & SPRING & AESTIVUM \\
\hline 23. & FEDERATION & FR & AUSTRALIA & AUS & SPRING & AESTIVUM \\
\hline 24. & FLORENCE & FLO & $?$ & ? & $?$ & $?$ \\
\hline 25. & FROCOR & FCR & BRAZIL & BRA & SPRING & AESTIVUM \\
\hline 26. & FUFAN17 & FFN & CHINA & $\mathrm{CHN}$ & SPRING & AESTIVUM \\
\hline 27. & FURY & FURY & KENYA & KEN & SPRING & AESTIVUM \\
\hline 28. & GABO-AUS & GB & AUSTRALIA & AUS & SPRING & AESTIVUM \\
\hline 29. & GAZA & GAZA & EGYPT & EGY & SPRING & DURUM \\
\hline 30. & GENERAL URQUIZA & GU & ARGENTINA & ARG & SPRING & AESTIVUM \\
\hline 31. & HARD FEDERATION & $\mathrm{HF}$ & AUSTRALIA & AUS & SPRING & AESTIVUM \\
\hline 32. & HARDRED CALCATTA & HRC & INDIA & IND & SPRING & AESTIVUM \\
\hline 33. & HOPE & $\mathrm{H} 44$ & USA & USA & $?$ & AESTIVUM \\
\hline 34. & HYBRID DELHI845 & HD845 & INDIA & IND & SPRING & AESTIVUM \\
\hline 35. & IUMILLO & IU & USA & USA & SPRING & DURUM \\
\hline 36. & KANRED & KR & USA & USA & WINTER & AESTIVUM \\
\hline 37. & KAVKAZ & KVZ & RUSSIA & RSA & WINTER & AESTIVUM \\
\hline 38. & KENTANA48 & KT48 & MEXICO & MEX & SPRING & AESTIVUM \\
\hline 39. & KENYA C6042 & КС6042 & KENYA & KEN & $?$ & $?$ \\
\hline 40. & KENYA GOVERNER & $\mathrm{KGV}$ & KENYA & KEN & SPRING & AESTIVUM \\
\hline 41. & KENYA STANDARD & $\mathrm{KS}$ & KENYA & $\mathrm{KEN}$ & SPRING & $?$ \\
\hline 42. & KENYA117A & K117A & KENYA & KEN & SPRING & AESTIVUM \\
\hline 43. & KENYA256 & $\mathrm{K} 256$ & KENYA & KEN & SPRING & $?$ \\
\hline 44. & KENYA291 & K291 & KENYA & KEN & SPRING & AESTIVUM \\
\hline 45. & KENYA324 & K324 & KENYA & $\mathrm{KEN}$ & SPRING & $?$ \\
\hline 46. & KENYA350-A-D9-C-2 & $\mathrm{KAD}$ & KENYA & KEN & SPRING & $?$ \\
\hline 47. & KENYA58 & K58 & KENYA & $\mathrm{KEN}$ & SPRING & AESTIVUM \\
\hline 48. & KHAPLI & KHP & INDIA & IND & SPRING & DURUM \\
\hline 49. & KLEIN ATLAS & KLAT & ARGENTINA & ARG & SPRING & AESTIVUM \\
\hline 50. & KLEIN RENDIDOR & KLRE & ARGENTINA & ARG & SPRING & AESTIVUM \\
\hline 51. & LA ESTANZUELA2787C & LAEST & $?$ & $?$ & ? & $?$ \\
\hline 52. & LC55 & LC55 & INDIA & IND & $?$ & $?$ \\
\hline 53. & LERMA ROJO & LR & MEXICO & MEX & SPRING & AESTIVUM \\
\hline 54. & MARNE DESPREZ & MD & FRANCE & FRA & WINTER & AESTIVUM \\
\hline
\end{tabular}




\begin{tabular}{|c|c|c|c|c|c|c|}
\hline \multirow[t]{2}{*}{ SN } & \multirow[t]{2}{*}{ Name } & \multirow[t]{2}{*}{$\mathrm{Abb} \dagger$} & \multicolumn{2}{|l|}{ Origin } & \multirow[t]{2}{*}{ Growth habit } & \multirow[t]{2}{*}{ Species } \\
\hline & & & Name & Abb & & \\
\hline 55. & MARROQUI & MRQ & MOROCCO & MAR & $\begin{array}{l}\text { SPRING } \\
\end{array}$ & AESTIVUM \\
\hline 56. & MARSALL'S NO 3 & MS-A & AUSTRALIA & AUS & $?$ & AESTIVUM \\
\hline 57. & MCMURACHY & MCM & CANADA & CAN & SPRING & AESTIVUM \\
\hline 58. & MIDA-U & MIDA & USA & USA & SPRING & AESTIVUM \\
\hline 59. & MUNDIA & MUNDIA & INDIA & IND & $?$ & $?$ \\
\hline 60. & NAINARI60 & NAI60 & MEXICO & MEX & $?$ & AESTIVUM \\
\hline 61. & NAPO & NAPO & COLOMBIA & $\mathrm{COL}$ & SPRING & AESTIVUM \\
\hline 62. & NARINO59 & NAR59 & COLOMBIA & $\mathrm{COL}$ & SPRING & AESTIVUM \\
\hline 63. & NEW PUSA773 & NP773 & INDIA & IND & SPRING & AESTIVUM \\
\hline 64. & NORIN10 & N10 & JAPAN & JPN & WINTER & AESTIVUM \\
\hline 65. & NP114 & NP114 & INDIA & IND & $?$ & AESTIVUM \\
\hline 66. & OLESEN'S DWARF & ON & ZIMBABWE & ZIM & SPRING & AESTIVUM \\
\hline 67. & P4160E & P4160E & MEXICO & MEX & SPRING & AESTIVUM \\
\hline 68. & POLYSSU & PSSU & BRAZIL & BRA & SPRING & AESTIVUM \\
\hline 69. & QUINTZEL & QTZ & $?$ & $?$ & $?$ & $?$ \\
\hline 70. & RED FIFE & $\mathrm{RF}$ & CANADA & CAN & SPRING & AESTIVUM \\
\hline 71. & RED MACE & RM & GREAT BRITAIN & GBR & WINTER & AESTIVUM \\
\hline 72. & REITI & REITI & $?$ & $?$ & $?$ & $?$ \\
\hline 73. & S339 & S339 & INDIA & IND & SPRING & AESTIVUM \\
\hline 74. & SANTA ELENA & SE & USA & USA & SPRING & AESTIVUM \\
\hline 75 . & SINVALOCHO MA & SCHOMA & ARGENTINA & ARG & SPRING & AESTIVUM \\
\hline 76. & SPALDING PROLIFIQUE & SPP & GREAT BRITIAN & GBR & $?$ & AESTIVUM \\
\hline 77. & STEINWEDEL & SWD & AUSTRALIA & AUS & SPRING & AESTIVUM \\
\hline 78. & TEZANOS PINTOS RECOZ & TZPP & ARGENTINA & ARG & SPRING & AESTIVUM \\
\hline 79. & THEW & THEW & AUSTRALIA & AUS & WINTER & AESTIVUM \\
\hline 80. & TIMESTEIN & $\mathrm{T}$ & AUSTRALIA & AUS & SPRING & AESTIVUM \\
\hline 81. & TYPE1 & TYPE1 & PAKISTAN & PAK & $?$ & DURUM \\
\hline 82. & TYPE9 & TYPE9 & PAKISTAN & PAK & $?$ & AESTIVUM \\
\hline 83. & VERNAL EMMER & VN & RUSSIA & RSA & SPRING & DURUM \\
\hline 84. & WAGGA13 & WG13 & $?$ & $?$ & $?$ & $?$ \\
\hline 85. & WEIQUE & W & DEUTSCHLAND & DEU & WINTER & AESTIVUM \\
\hline 86. & WILHELMINE & WHM & NETHERLANDS & NLD & WINTER & AESTIVUM \\
\hline 87. & WILLET ERONO & WTE & USA & USA & SPRING & AESTIVUM \\
\hline 88. & WIS 245 & WIS 245 & $?$ & $?$ & $?$ & $?$ \\
\hline 89. & YAKTANA54 & YT54 & MEXICO & MEX & SPRING & AESTIVUM \\
\hline
\end{tabular}

$\dagger$ Abb, Abbreviation.? Not known.

Use of 89 ancestors from 22 different countries represent the great diversity in built in 35 Nepalese wheat cultivars. Ancestors were with different growth habit eg spring (57.31\%) and winter (13.48\%). There were $76.40 \%$ aestivum and 5.62\% durum ancestors species. Such diversity in species, origin and growth habit in ancestors have certainly enriched Nepalese wheat biodiversity.

Despite high yielding attributes of improved cultivars compared to those of local types improved varieties were found to be susceptible to diseases and lodging (Shrestha 1976). There are many examples of improved varieties gaining popularity within a short period of time but later become susceptible to biotic stresses. Such trend was not reported in landraces. In this study, cultivars surveyed represent a wide range of variation for different areas of origin and adaptation. This genetic diversity may be useful for further wheat improvement. The results of this study may help in the selection of the most diverse cultivars and greatly expand genetic variation for wheat improvement. Measurers of genetic diversity can be used to maximize the level of variation in segregating populations by intermating cultivars with greater genetic distance. Modern varieties are replacing the landraces and improved old varieties resulted in the genetic erosion. Therefore, in situ, on farm and ex situ conservations are necessary for maintaining the genetic variation in Nepalese wheat. Government, semi 
governmental and private agencies should take action to conserve and utilize wheat genetic variations present in Nepal.

Nepal being proximal to the secondary source of origin of wheat, might harbor the wheat relatives in addition to the so far recorded one species of Aegiolopes and nine species of Agropyron. Since wild germplasm is a valuable source for improving bread wheat productivity and durability, explorations on those wild relatives are suggested followed by their proper characterization and utilization in the breeding program. Gene pool from cultivated landraces of spring and winter type, wild landraces and diploid species of wheat along with international gene pool could make to success in developing high yielding cultivars with wide adaptability.

\section{ACKNOWLEDGEMENTS}

Sincere thanks go to GO Ferrera and HB KC for providing literatures. S Rai provided e-maps and TP Pokhrel suggested for improving this paper.

\section{REFERENCES}

Bhatta MR, GO Ferrera, B Gurung, TP Pokharel, NR Gautum, P Gurung and RB Neupane. 2000. Present status of participatory plant breeding research on wheat at the National Wheat Research Programme, Nepal. In: $A n$ exchange and experiences from South and South East Asia: Proceedings of the International Symposium on PPB and PGR enhancement, Pokhara, Nepal, 1-5 May 2000. PRGA, IDRC, DFID, DDS, LIBIRD, IPGRI and ICARDA. Pp. 391-398.

Bland B. 2001. Nepalese wheat pool. In: The world wheat book: A history of wheat breeding. (AP Bonjean and WJ Angus, eds). Intercept, TEC and DOC, LAVOISIER, New York. Pp. 817-830.

Cox TS, YT Kiang, MB Gorman and DM Rogers. 1985a. Relationship between coefficient of parentage and genetic similarity indices in the soybean. Crop Sci. 25:529-532.

Cox T, GL Lokhart, DE Walker, LG Harrell, LD Albers and DM Rogers. 1985b. Genetic relationships among hard red winter wheat cultivars as evaluated by pedigree analysis and gliadin polyacrylamide gel electrophoretic patterns. Crop Sci. 25:1058-1062.

Dilday RH. 1990. Contribution of ancestral lines in the development of new cultivars of rice. Crop Sci. 30:905911.

Gupta SR, MP Upadhyay and DM Dongol (eds). 2000. Nepalese germplasm catalogue - 2000. Agriculture Botany Division, NARC, Khumaltar.

Hawkes J. 1981. Germplasm collection, preservation and use. In: Plant Breeding II (Frey KJ, ed.). The Iowa State University Press/ Ames.

Jain KBL. 1994. Wheat cultivars in India: Names, pedigrees, origins, and adaptations. Research Bulletin No. 2. Directorate of Wheat Research, Karnal. India. $72 \mathrm{pp}$.

Joshi BK, A Mudwari, MR Bhatta and GO Ferrera. 2004. Genetic diversity in Nepalese wheat cultivars based on agro-morphological traits and coefficients of parentage. Nepal Agric. Res. J. 5:7-18

Martin JM, TK Balke and EA Hockett. 1991. Diversity among North American spring barley cultivars based on coefficients of parentage. Crop Sci. 31:1131-1137.

Mudwari A. 1999. Wild relatives of wheat and its status in Nepal. In: Wild relatives of cultivated plants in Nepal (R Shrestha and B Shrestha, eds). Proceedings of National conference, 2-4 June 1999 Kathmandu, GEMNepal. Pp.83-89

NARC. 1997. 25 years of wheat research in Nepal (1992-1997). NARC, NWRP, Nepal.

Shrestha RB. 1976. Wheat production technology in Nepal. Nepalese Journal of Agriculture 6:18-24.

Skovmand B, MC Mackary, C Lopez and A McNab (eds). 2000. Tools for the millennium. On compact disk, Mexico, D.F.: CIMMYT.

Skovmand B, R Vilereal, M Van Ginkel, S Rajaram and GO Ferrera. 1997. Semi dwarf bread wheats: Names, parentages, pedigrees and origins. Mexico. D.F.: CIMMYT.

Souza, E and ME Sorrells. 1989. Pedigree analysis of North American oat cultivars released from 1951 to 1985. Crop Sci. 29:595-601. 